Special Issue of the 6th International Congress \& Exhibition (APMAS2016), Maslak, Istanbul, Turkey, June 1-3, 2016

\title{
Analysis of Radial Dependence of the Localized Magnetic Field using Artificial Neural Networks
}

\author{
A.H. ISIK ${ }^{a, *}$ AND N. ISIK ${ }^{b}$ \\ ${ }^{a}$ Mehmet Akif Ersoy University, Department of Computer Engineering, 15030 Burdur, Turkey \\ ${ }^{b}$ Mehmet Akif Ersoy University, Department of Science Education, 15030 Burdur, Turkey
}

\begin{abstract}
The measurements of the angular distributions of charged particles have a long history in atomic and molecular collision studies. To detect all electrons originating from collision has great importance in experimental studies. Due to the physical constraints of the experimental instruments, electrons in definite angles can be detected. Magnetic angle changer is designed to steer electrons scattered at undetectable angles. The magnetic angle changer is a source of the localized magnetic field. A well-controlled magnetic field in the interaction region changes the angles of the electron trajectories. In this study, artificial neural networks have been performed to obtain variation of the magnetic field strength as a function of radial distance calculated from boundary element method. A stringent quality filter is used for data to produce more robust artificial neural network based prediction. The results indicate that the well-trained artificial neural networks can predict the effect on the radial dependence of the localized magnetic field with tremendous precision. It is believed that this study will introduce a new insight into collision studies.
\end{abstract}

DOI: 10.12693/APhysPolA.131.32

PACS/topics: 42.79.Fm, 07.05.Tp, 07.77.-n

\section{Introduction}

Detection of over the full range of scattering electrons is an outstanding challenge in a wide range of experimental measurements. In particular, the magnetic angle changer (MAC) device provides a powerful tool for the detection of over the full range of scattering electrons for electron-electron coincidence measurements [1-8]. The MAC device is combined by a pair of counterpropagating coils. By combining the localized magnetic field, the deflection of electrons at the desired angles becomes possible [9-11].

In recent years, artificial neural networks have seen an explosion of interest in atomic and molecular physics [12-16]. Artificial neural networks (ANNs) are parallel computing systems which are modelled on the behavior of neurons in the human brain. In this study, the ANN method is employed to predict the magnetic field originating from the MAC device as a function of radial distance from the center of the MAC coils. By using ANN method, the electron paths can be determined on time scales as short as two seconds.

The work is organized as follows. Section 2 includes the detailed information about magnetic angle changer, Sect. 3 presents fundamental and distinguish features of nonlinear autoregressive exogenous model (NARX) based ANN method. Test results of the trained network with obtained data using $\mathrm{CPO}$ simulation program are given in Sect. 4.

*corresponding author; e-mail: ahakan@mehmetakif.edu.tr

\section{Magnetic angle changer}

MAC device is originally designed for changing the angles of electron trajectories by Read and Channing [9]. With this design, the paths of electrons are changed by the generated magnetic field. The device consists of a set of solenoids located around the interaction region. It is given in Fig. 1. Through the solenoids, the MAC introduces a localized well-localized magnetic field at the interaction region. It is necessary to ensure that the electrons outside the solenoids are not affected by the magnetic field. This is achieved by the determination of the variation of the magnetic field as a function of radial distance.

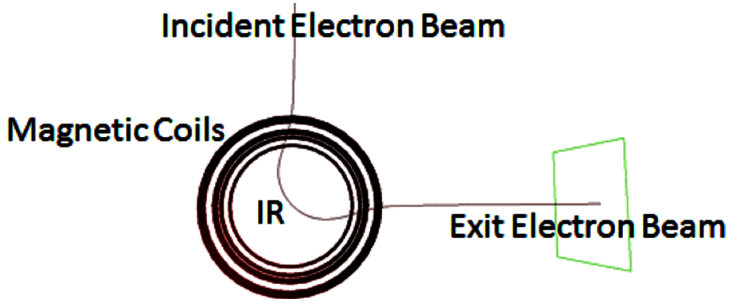

Fig. 1. Representation of the paths of electrons in the MAC field. IR - interaction region.

In this study, the prediction results of the radial dependence of the localized magnetic field are obtained with ANN method.

\section{ANN method}

Inspired by the biological neural system architecture, ANNs have the ability of learning and generalization. 
This heuristic algorithm imitates human behavior. ANNs consist of the large number of interconnected neurons. Through these mathematical neurons, a weighted sum of the input variables is computed and mathematical operation is performed. After these operations, output and error value is found. Error is the difference between real and obtained value. Depending on the quality and number of training data and learning model, prediction performance can be improved. In this study, we used NARX model ANN. Designed ANN learns the radial dependence of the localized magnetic field.

\section{Results}

To explore the radial dependence of the localized magnetic field generated using MAC device, we systematically analyzed the training data obtained by the CPO program. In first step process, the dataset is filtered for the best prediction. Jointly, these dataset is normalized to the range of -1 to +1 . Remarkably, our results demonstrate that the normalization technique improved the performance results. The 86 samples were used in artificial neural network prediction technique. The dataset were divided into $70 \%$ training set, $15 \%$ validation set and $15 \%$ test set. We trained the network with these refined dataset. The aim of training is to find optimum values for the weights connecting the input, hidden and output layers. In training period, we used LevenbergMarquardt backpropagation algorithm in ANN. In addition, different ANN architectures were performed by changing the number $N$ of hidden neurons.

MSE results for NARX model.

TABLE I

\begin{tabular}{c|c|c}
\hline \hline$N$ & Training & Test \\
\hline 5 & $2.52 \times 10^{-10}$ & $6.17 \times 10^{-6}$ \\
10 & $4.02 \times 10^{-10}$ & $2.67 \times 10^{-5}$ \\
15 & $1.83 \times 10^{-7}$ & $1.52 \times 10^{-4}$ \\
20 & $2.52 \times 10^{-11}$ & $1.96 \times 10^{-9}$
\end{tabular}

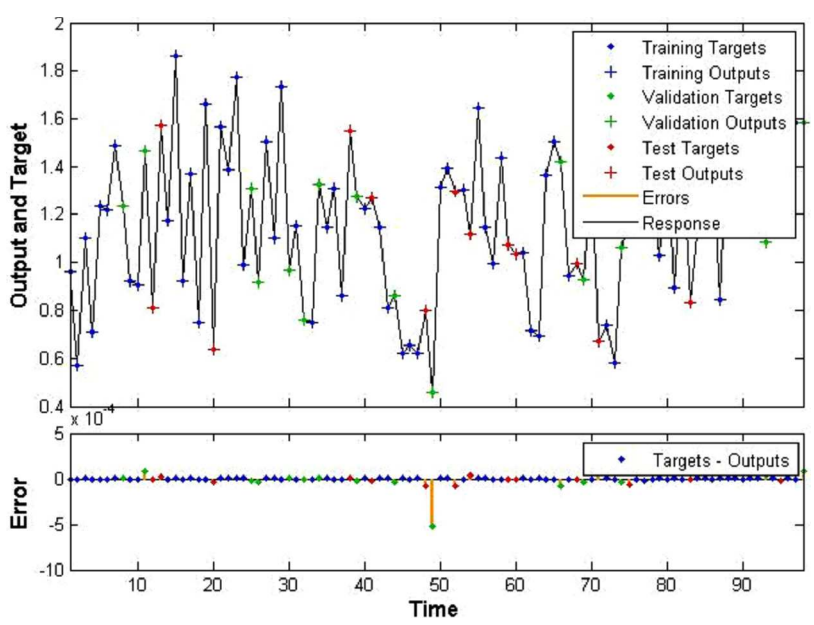

Fig. 2. Time series response of NARX.
The performance results with the mean square error (MSE) values for different architectures were summarized in Table I. In every case regression level $R=0.999$. Presented results demonstrated that 2-20-2 ANN architecture predicts with tremendous precision.

In Fig. 2, time series response of output element is given for 2-20-2 ANN architecture. It is seen that, NARX based time series ANN method has minimum error.

\section{Conclusion}

Our results show that ANN method is a practical method to find the electron trajectories in MAC. This study is also exemplary in demonstrating the application to problems of prediction of electron beam trajectories. Obtained performance results show that ANN is an innovative and high precision method. In the future, different types of heuristic algorithms can also be used in this field.

\section{Acknowledgments}

This research has been supported by Mehmet Akif Ersoy University Scientific Projects. Project numbers: NAP-0337-16 and NAP-0296-16.

\section{References}

[1] D. Cubric, D.B. Thompson, D.R. Cooper, G.C. King, F.H. Read, J. Phys. B At. Mol. Opt. Phys. 30, 857 (1997).

[2] B. Mielewska, G.C. King, F.H. Read, M. Zubek, Chem. Phys. Lett. 311, 428 (1999).

[3] H. Cho, H.S. Lee, Y.S. Park, Radiat. Phys. Chem. 68, 115 (2003).

[4] A. Murray, M. Hussey, W. MacGillivray, G. King, AIP Conf. Proc. 811, 179 (2006).

[5] L. Klosowski, M. Pivinski, D. Dziczek, K. Wisniewska, S. Chiwirot, Meas. Sci. Technol. 18, 3801 (2007).

[6] M. Hussey, A.J. Murray, W. MacGillivray, G. King, N. Bowring, J. Phys. Conf. Ser. 88, 012061 (2007).

[7] M. Stevenson, B. Lohmann, J. Electron. Spectrosc. Relat. Phenom. 161, 31 (2007).

[8] M. Hussey, A.J. Murray, W. MacGillivray, G. King, J. Phys. B At. Mol. Opt. Phys. 41, 055202 (2008).

[9] F.H. Read, J.M. Channing, Rev. Sci. Instrum. 67, 2372 (1996).

[10] B. Mielewska, Radiat. Phys. Chem. 76, 418 (2007).

[11] D. Cubric, D.J.L. Mercer, J.M. Channing, G.C. King, F.H. Read, J. Phys. B At. Mol. Opt. Phys. 32, 45 (1999).

[12] A.H. Isik, Acta Phys. Pol. A 127, 1317 (2015).

[13] A.H. Isik, Acta Phys. Pol. A 127, 1717 (2015).

[14] N. Isik, Microsc. Microanal. 22, 458 (2016).

[15] N. Isik, A.H. Isik, Acta Phys. Pol. A 129, 628 (2016).

[16] A.H. Isik, N. Isik, Acta Phys. Pol. A 129, 514 (2016). 